\title{
LA POSICIÓN DE LOS ESTADOS MIEMBROS EN EL TRATADO CONSTITUCIONAL EUROPEO
}

\author{
POR \\ JAVIER TAJADURA TEJADA \\ Profesor Titular de Derecho constitucional \\ Universidad del País Vasco
}

\section{LA NATURALEZA JURÍDICA DE LA UNIÓN EUROPEA: UNA CONFEDERACIÓN DE ESTADOS EN SU FORMA MODERNA}

Europa se presenta ante el jurista como una organización de Estados, cuya verdadera naturaleza jurídica resulta difícil de determinar y por ello controvertida. En todo caso, lo que resulta indiscutible es que dicha organización se inspira y se vertebra sobre los valores y principios del federalismo. La Unión Europea presenta numerosos rasgos propios de un esquema federal de articulación del poder: las instituciones comunitarias ejercen directamente potestades normativas y jurisdiccionales sobre los particulares; existe un orden de distribución de competencias entre la Unión y los Estados miembros; los mecanismos de resolución de conflictos encajan también en el esquema federal. Admitido esto, el problema surge cuando se trata de determinar a cuál de las manifestaciones estructurales históricas de éste se adscribe la nueva comunidad política europea. Y ello porque la Unión Europea tal y como está actualmente configurada por el Tratado de Niza, y tal y como viene definida por el TICE, no responde de manera total, plena y absoluta ni al concepto clásico del Estado Federal ni al de Confederación de Estados. La Unión Europea presenta rasgos y elementos de uno $\mathrm{y}$ de otra. 
Entre las preocupaciones principales de la Convención que ha redactado el PTICE ${ }^{1}$, no ha figurado nunca la de fijar o establecer una manifestación concreta del fenómeno federal. La razón no debe ser difícil de comprender. Tal propósito hubiera fracasado ante posiciones tan divergentes como las defendidas por Francia, Alemania y el Reino Unido. En este sentido, debemos destacar que la Convención, cuando redactó el PTICE, no estaba pensando, ni tenía por qué, en fijar una manifestación concreta del federalismo equiparable a alguno de los modelos que la historia nos proporciona. Su objetivo era estructurar Europa mediante una organización política que respondiera a las necesidades de las poblaciones de los Estados miembros. Es a los teóricos a los que "a posteriori" nos corresponde construir los modelos induciéndolos de las decisiones eminentemente prácticas que los negociadores del Tratado, en virtud de sus compromisos, se han visto obligados a adoptar. Esto quiere decir que los modelos sólo existen en la mente de los teóricos que los construyen. Estos modelos o "tipos ideales" como les Ilamaría Max Weber son fruto de la necesidad de poner un cierto orden conceptual en una realidad que es infinitamente compleja.

Con clarividencia advirtió esto don Manuel Azaña en el tan célebre como poco leído discurso "España ha dejado de ser católica", pronunciado en la sesión de Cortes de 13 de octubre de 1931. Su discurso sobre la relación entre los problemas reales de España y la configuración de su organización política, es extrapolable al contexto europeo: "Realidades vitales (...); esto es lo que debemos llevar siempre ante los ojos; realidades vitales que son antes que la ciencia, que la legislación y que el gobierno, y que la ciencia, la legislación y el gobierno acometen y tratan para fines diversos y por métodos enteramente distintos. La vida inventa y crea; la ciencia procede por abstracciones que tienen una aspiración, la del valor universal; pero la legislación es, por lo menos, nacional y temporal, y el gobierno - quiero decir el arte de gobernares cotidiano. Nosotros debemos proceder como legisladores y como gobernantes y hallar la norma legislativa y el método de gobierno que nos permita resolver las antinomias existentes en la realidad (...) de

1 EI TICE consta de cuatro partes. La Parte Primera contiene la arquitectura constitucional de la Unión, con nueve títulos relativos a los objetivos, las instituciones, las competencias, la tipología de actos normativos y procedimientos decisorios, las finanzas, la acción exterior y la pertenencia a la Unión; la Parte Segunda se dedica a la protección de los derechos fundamentales e incluye, con algún cambio, el contenido de la Carta de Derechos Fundamentales; la Parte Tercera se refiere a las políticas y a la realización de acciones de la Unión, e incorpora, con ligeras modificaciones, el conjunto del derecho material de la Unión; finalmente, la Parte Cuarta contiene una serie de disposicions generales y finales. 
hoy; después vendrá la ciencia y nos dirá cómo se llama lo que hemos hecho" ${ }^{2}$.

Por ello, la primera cuestión que hemos de afrontar es la relativa a la naturaleza de la Unión Europea, a la que por primera vez el TICE le atribuye personalidad jurídica ${ }^{3}$.

Ubicado el proceso de integración europea en la senda del federalismo, veamos las razones que explican la dificultad de situar con claridad a la Unión Europea bien en la órbita del Estado Federal, bien en la de la Confederación de Estados.

Para estos efectos y siguiendo al profesor Ruipérez ${ }^{4}$, debemos recordar que fue Georg Meyer, entre todos los teóricos del federalismo, el que formuló la distinción entre las dos manifestaciones del fenómeno federal de la forma más tenue y menos intensa habida cuenta que, según el, la celebración del Tratado por el que se crea la Confederación de Estados supone ya la renuncia por parte de sus integrantes a su status de Estados soberanos e independientes, sujetos del Derecho Internacional, en beneficio de la nueva entidad política. Confederación y Estado Federal quedan así, aparentemente, equiparados. Y digo, aparentemente, porque para G. Meyer dos fundamentales diferencias impiden llevar a cabo la mencionada equiparación. En primer lugar, el procedimiento en virtud del cual puede verificarse la reforma de su norma fundacional es diferente según nos encontremos en un Estado Federal o en una Confederación. Mientras que en la Confederación la reforma de su texto fundamental requiere, con carácter general, la unanimidad de los miembros, en el caso del Estado Federal, es suficiente con que el proyecto de revisión sea aprobado por una mayoría cualificada. Y, en segundo lugar, diferentes son también los efectos que las normas jurídicas emanadas de los órganos centrales producen sobre los ciudadanos según nos encontremos en un Estado Federal o en una Confederación. En el Estado Federal, las normas jurídicas de la Federación son directamente aplicables a todos los ciudadanos y no necesitan de ninguna convalidación por parte de las autoridades de las colectividades miembros. En la Confederación, por el contrario, las normas confederales sólo pueden ser aplicadas a los ciudadanos en la medida en que han sido transformadas en derecho interno por las autoridades de las colectividades miembros.

2 Azaña, M.: "Discursos políticos» (edición de Santos Juliá), Crítica, Barcelona, 2003, pp. 113 y 114.

3 Artículo 6 del TICE: "La Unión tendrá personalidad jurídica».

4 RuIPÉREZ, J.: La "Constitución europea" y la problemática del Poder Constituyente, Biblioteca Nueva, Madrid, 2000, pp. 45 y ss. 
Atendiendo a la primera diferencia, y como expondremos después, la Unión Europea en la medida en que sus normas fundacionales deben ser siempre reformadas por unanimidad, se situaría en la órbita de la Confederación de Estados. Pero si nos atenemos a la segunda de las diferencias acuñadas por la clásica Teoría del Estado, la Unión Europea dada la aplicación preferente y directa de sus normas en el seno de los Estados miembros estaría mucho más próxima a la forma de Estado Federal.

Son las anteriores consideraciones las que nos llevan a compartir las tesis de los profesores La Pérgola o Ruipérez, en el sentido de afirmar la necesidad de reformular las categorías clásicas sobre el federalismo de la Teoría del Estado ${ }^{5}$. De lo que se trataría es de diferenciar dentro de la Confederación entre una forma antigua o arcaica y una forma moderna. A la primera responderían los modelos históricos por todos conocidos: la Unión de las trece antiguas colonias americanas desde el 5 de noviembre de 1779 hasta la puesta en marcha de la Convención de Filadelfia; la Confederación Helvética desde 1803 a 1848; o la situación de los Lánder alemanes hasta la creación del Imperio guillermino y la aprobación de la Constitución de 1871. A la segunda opción se adscribiría la Unión Europea. "Su principal característica - escribe el profesor Ruipérez-sería la de que no siendo aún un Estado Constitucional en su manifestación de Estado Federal, se encuentra mucho más centralizado de lo que lo estuvieron las Confederaciones históricas» ${ }^{6}$.

\section{LAS COMPETENCIAS DE LOS ESTADOS MIEMBROS EN EL TICE}

La Unión Europea se configura, por tanto, como una Confederación de Estados en su forma moderna. Por ello, de la misma manera que ocurría en su manifestación arcaica, la Unión Europea se presenta como una nueva entidad política distinta de cada uno de sus componentes, dotada de personalidad jurídica internacional y de una organización institucional propia para el ejercicio de sus competencias.

En este contexto, y como ha advertido el profesor Ruipérez en su brillante y sugerente monografía sobre el proceso de constitucionalización de Europa, el primer problema al que, inexcusablemente, habrá

5 La Pergola, A.: Los nuevos senderos del federalismo, CEC, Madrid, 1994, pp. 124-138; 151-190. RuIPÉREZ, J.: "Algunas cuestiones sobre el momento estatal de la integración europea: La Unión Europea como forma moderna de Confederación (una primera aproximación)" en El constitucionalismo en la crisis del Estado Social (García Herrera, M. A., dir.), Universidad del País Vasco, Bilbao, 1997, pp. 619-645.

6 RuIPÉREZ, J.: La "Constitución europea»..., ob.cit., p. 49. 
de enfrentarse el constitucionalista, no es otro que el de determinar cuál es el status jurídico que tiene el viejo Estado nacional en el marco de la Unión Europea ${ }^{7}$. Y ello porque, como con meridiana claridad y acierto pleno ha subrayado el profesor Díez Picazo, "toda discusión sobre la constitucionalización de la Unión Europea ha de ser consciente de en qué medida sus Estados miembros siguen siendo auténticos Estados" ${ }^{8}$.

A ello se dedican las páginas que siguen. Como vamos a ver, del estudio del reparto de las competencias entre los Estados miembros y la Unión establecido en el TICE no cabe deducir, en modo alguno, que la Unión Europea haya abandonado su fundamento jurídico internacional y haya dejado de ser una Unión de Estados o lo que hemos denominado de la mano de los profesores La Pérgola y Ruipérez, Confederación en su forma moderna. Y ello por la sencilla razón de que los Estados miembros conservan las facultades y competencias inherentes a su condición de Estados soberanos.

El artículo 5 del TICE se configura, en este sentido, como el inexcusable punto de partida para el análisis de esta cuestión. Dicho precepto lleva por rúbrica "relaciones entre la Unión y los Estados miembros" y consagra el principio del respeto a la identidad nacional de aquellos:

"La Unión respetará la identidad nacional de los Estados miembros, inherentes a las estructuras fundamentales políticas y constitucionales de éstos, también en lo que respecta a la autonomía local y regional. Respetará las funciones esenciales del Estado, en particular las que tienen por objeto garantizar su integridad territorial, mantener el orden público y salvaguardar la seguridad interior».

Con estas premisas y dado que el Estado, como una unidad de acción y de decisión políticas (Heller), es definido mediante tres elementos constitutivos: el aparato de gobierno (poder), la población y el territorio, debemos examinar, en primer lugar, a quien corresponden, si a la Unión o a los Estados miembros, las competencias fundamentales relativas al establecimiento de los modos y formas de organización política y a la delimitación del ámbito territorial y personal de la Unión. Analizaremos después las competencias para ejecutar medidas de coerción y, finalmente, la decisiva facultad que conservan los Estados miembros, de abandonar la Unión.

7 RUIPÉREZ, J.: La «Constitución europea»..., ob. cit., pp. 52-53.

8 Díez PicAzo, L. M.: Constitucionalismo de la Unión Europea, Civitas, Madrid, 2002, p. 169. 


\subsection{La competencia de autoorganización político-constitucional. El principio de autonomía institucional.}

El artículo 57 del TICE establece que la Unión estará abierta a todos los Estados europeos que respeten los valores mencionados en el artículo 2 y se comprometan a promoverlos en común. Se fijan así cuáles son las condiciones o requisitos que un Estado debe cumplir para poder adherirse a la Unión Europea. Los candidatos al ingreso deben ser en primer lugar y desde un punto de vista estrictamente geográfico aunque no por ello aproblemático, Estados europeos; y en segundo lugar, debe tratarse de Estados que asuman un concreto orden material de valores: "respeto a la dignidad humana, libertad, democracia, igualdad, Estado de Derecho y respeto a los derechos humanos». En otros contextos, probablemente, el contenido del artículo 57 podría resultar ambiguo o indeterminado, pero en el caso de la Unión Europea, su significado y alcance resultan meridianamente claros. Sólo pueden formar parte de la Unión Estados constitucionales democráticos. Tal condición democrática se presume respecto de todo Estado admitido en el Consejo de Europa. Y, aunque es preciso reconocer que, desde 1989, los criterios de homologación del Consejo de Europa han sufrido una peligrosa relajación, no debe quedar ninguna duda de que si un Estado no supera el test de efectividad respeto a los principios de la democracia constitucional no puede ingresar en la Unión, y si está ya dentro puede ser, sancionado con la suspensión en sus derechos ${ }^{9}$.

En todo caso, lo que nos interesa subrayar es que a salvo lo anterior, la pertenencia a la Unión Europea no prejuzga ninguna otra cuestión relativa a los sistemas constitucionales de los Estados miembros ${ }^{10}$. Dicho con otras palabras, el artículo 57 deja un amplísimo espacio de configuración política a los Estados miembros. La Unión Europea nada tiene qué decir sobre si la forma de la jefatura del Estado es monárquica o republicana, sobre si la relación entre los poderes del Estado se articula sobre los esquemas del parlamentarismo o del presidencialismo, sobre si se reconoce o no el derecho a la autonomía política de entidades infraestatales, sobre si la judicatura debe ser funcionarial o no, sobre si la justicia constitucional debe articularse de forma concentrada

9 Informe de 8 de setiembre de 2000, elaborado por Martti Athisaari, Jochen Frowein y Marcelino Oreja, sobre la observancia por parte de Austria de las condiciones de pertenencia a la Unión Europea, tras el acceso de la extrema derecha al Gobierno de la República, Revista de Derecho Comunitario Europeo, n. ${ }^{\circ}$ 8, 2000.

10 DíEZ PICAZO, L. M.: “¿Qué significa ser Estado dentro de la Unión Europea?» en La encrucijada constitucional de la Unión Europea (E. García de Enterría, dir.), Civitas, Madrid, 2002, pp. 270 y ss. 
o difusa, etc. Todas las anteriores son opciones legítimas dentro de una Democracia Constitucional, por lo que en el seno de la Unión caben sistemas políticos diferentes.

En conclusión, los Estados miembros conservan plenamente su capacidad autoorganizativa para configurar sus propios sistemas políticos. Esta libertad de autoorganización se denomina en el derecho comunitario, "principio de autonomía institucional»" ${ }^{11}$ y es algo que no encaja en los esquemas federales.

Establecido lo anterior, sólo resta señalar que según reiterada jurisprudencia del Tribunal de Justicia, el principio de autonomía institucional se encuentra sometido a dos límites. En su sujeción al derecho comunitario, los Estados miembros a la hora de aplicar las normas comunitarias son libres de hacerlo mediante las fórmulas organizativas y procedimentales que consideren más oportunas, pero están sujetos a esta doble limitación: primero, los procedimientos administrativos y judiciales deben ser efectivos; segundo, dichos procedimientos no pueden ser más gravosos que aquellos que se aplican a asuntos similares de derecho puramente interno. Son estos límites los que explican el carácter problemático del principio de autonomía institucional en relación

11 Importa subrayar que se trata de un principio que aunque pueda ser deducido de los ordenamientos nacionales (lo que los Estados no han transferido a la Unión permanece en la esfera de sus competencias, e, indiscutible resulta, que los Estados no han transferido la competencia de autoorganización constitucional y administrativa) es también un principio del propio ordenamiento comunitario con las consecuencias que ello implica. Efectivamente, como tal principio del ordenamiento comunitario fue reconocido por la Sentencia International Fruit Company c. Produktschap voor Groenten en Fruit, de 13 de mayo de 1971, que afirmó lo siguiente: "Cuando las disposiciones del Tratado o de reglamentos reconocen poderes o imponen obligaciones a los Estados miembros a efectos de la aplicación del derecho comunitario, la cuestión de saber de qué manera el ejercicio de esos poderes y la ejecución de esas obligaciones pueden ser encomendados por los Estados a determinados órganos depende únicamente del sistema constitucional de cada Estado». La "autonomía institucional» no puede por tanto ser entendida como una mera clausula residual en el sentido de que los Estados pueden autoorganizarse en la medida en que no haya norma comunitaria al respecto, sino que se configura como un principio que limita y condiciona la validez de los actos comunitarios. Esto es, una norma de derecho comunitario derivado que restringiera esa poder de autoorganización de los Estados no sería válida. Sobre el significado y alcance de este principio de autonomía institucional, RIDEAU, J.: Droit institutionnell des Communautés européennes, 2. ${ }^{a}$ ed., LGDJ, París, 1996, pp. 727 y ss. En la doctrina española, PÉREZ TREMPS, P.: "Las fuentes internacionales y supranacionales" en López Guerra, L. y otros, Derecho Constitucional, vol. I, 4. a ed., Tirant lo Blanch, Valencia, 2000, pp. 125 y ss. 
con dos temas centrales del Derecho constitucional: la independencia judicial y la autonomía política de las entidades infraestatales. En ambos supuestos el problema es idéntico y se circunscribe a cómo asegurar la aplicación efectiva del derecho comunitario por parte de determinadas autoridades de los Estados miembros que por imperativo constitucional actúan con independencia de su respectivo Gobierno. Es decir, de autoridades inmunes al poder de dirección de los órganos gubernativos a los que corresponde relacionarse con la Unión Europea. La respuesta del Tribunal de Justicia ha permitido cohonestar la autonomía institucional del Estado y la aplicación efectiva del Derecho Comunitario. Su bien conocida tesis puede resumirse así: la pertenencia a la Unión Europea no condiciona ni altera las opciones constitucionales de los Estados miembros, pero éstos no pueden invocar sus disposiciones constitucionales para intentar justificar el incumplimiento de obligaciones comunitarias, ni para eludir las responsabilidades que de ello pudiera derivarse ${ }^{12}$.

\subsection{La competencia sobre la delimitación del territorio}

El ordenamiento comunitario regula su propio ámbito espacial en una disposición del TICE. Se trata del artículo IV-4. La lectura del mismo nos pone de manifiesto que se trata de un precepto extenso y prolijo que, tras afirmar que el Tratado será aplicado a todos y cada uno de los Estados miembros, los cuales son a su vez enumerados, establece toda una serie de aclaraciones y excepciones con respecto a territorios concretos. Las razones de ello son fácilmente comprensibles: en primer lugar, porque por razones históricas bien conocidas, el territorio de diversos Estados miembros se extiende a lugares situados geográficamente fuera de Europa; y, en segundo lugar, porque dentro del propio ámbito geográfico de Europa, ciertos Estados miembros tienen parte de su territorio sometido a regímenes jurídicos especiales. En este sentido, la importantísima función que el art. IV-4 TICE cumple no es otra que determinar si el derecho comunitario se aplica o no, o, en su caso, con que particularidades, a cada uno de los territorios en él mencionados. Dicho esto, a los efectos de esta exposición, lo que nos interesa subrayar y destacar es que la definición que el citado precepto hace del ámbito de aplicación espacial del derecho comunitario depende, ex-

12 En relación a la independencia judicial, Sentencia Comisión c. Italia de 13 de diciembre de 1991. En relación a la autonomía política de entidades infraestatales, Sentencias Fratelli Costanzo c. Comune di Milano, de 22 de junio de 1989 y Comisión c. Alemania, de 12 de julio de 1990. 
clusivamente, de la definición del territorio de todos y cada uno de los Estados miembros en cuanto sujetos de derecho internacional. Dicho con otras palabras, la definición del territorio de los Estados miembros es una competencia exclusiva de éstos, y escapa, por completo, a la competencia normativa de los órganos de la Unión Europea. El territorio de la Unión Europea queda así definido por remisión: será la suma de lo que en cada momento sean los territorios de los Estados miembros. Estados que en cuanto sujetos de Derecho Internacional podrán ver aumentado o disminuido su territorio de conformidad con las correspondientes normas internacionales. Lo cual no deja de tener importantes repercusiones para la estructura de la Unión Europea. Toda adquisición o pérdida territorial de un Estado miembro repercute automáticamente sobre la Unión Europea, cuyos órganos, carecen de cualquier competencia sobre el particular. Esto quiere decir que el ámbito espacial de la Unión Europea se reduce o amplia sin necesidad de reformar las normas fundacionales. Conclusiones estas confirmadas por los hechos. A estos efectos conviene recordar los siguientes extremos:

a) Sin lugar a dudas, el caso más paradigmático de lo que acabamos de afirmar vino dado por la reunificación de Alemania el 3 de octubre de 1990. La incorporación de la República Democrática de Alemania, en forma de nuevos Lánder a la República Federal de Alemania, provocó la inmediata inclusión de la antigua Alemania Oriental en la Unión Europea ${ }^{13}$.

b) La independencia de Argelia en 1962 determinó también que el territorio del nuevo Estado, y que hasta entonces había pertenecido a la República Francesa, quedara automáticamente excluido del ámbito de aplicación espacial del ordenamiento comunitario.

Junto a los mencionados supuestos de incremento o disminución territorial de la Unión Europea, por incremento o disminución del territorio de alguno de sus Estados miembros, hay que señalar que aqueIlos pueden tener lugar también cuando un Estado modifica unilateralmente el régimen o estatuto jurídico de una parte de su territorio. Así, la isla de St. Pierre et Miquelon, perteneciente a la República Francesa, dejó de pertenecer a la Unión Europea en 1985, cuando el gobierno francés modificó su estatuto político-administrativo transformando el antiguo departamento en territorio de ultramar. $Y$ ello por la sencilla ra-

13 Sobre el procedimiento constitucional seguido para la reunificación alemana, ARNold, R.: La unificación alemana (Estudios sobre derecho alemán y europeo), Civitas, Madrid, 1993. 
zón de que el artículo 299 TCE preveía la aplicación del derecho comunitario en los departamentos franceses de Ultramar, pero no así en sus territorios de Ultramar.

Una mención especial requiere la retirada de Groenlandia operada mediante el Tratado de 13 de marzo de 1984, adoptado de conformidad con los procedimientos de revisión de los Tratados fundacionales ${ }^{14}$. Groenlandia era y es territorio del Reino de Dinamarca y no ha sufrido cambio alguno en su estatuto jurídico-administrativo. Sin embargo, por voluntad de sus habitantes se adoptó el mencionado Tratado que prevé la exclusión de Groenlandia de la Unión Europea. Fácilmente se comprende que, en este caso, la restricción del ámbito de aplicación espacial del derecho comunitario requiriese una modificación del Tratado fundacional. $Y$ es que, aquí, no nos encontramos, ni ante un territorio que se independiza, ni ante un territorio que modifica su estatuto político-administrativo, sino ante un supuesto sustancialmente diferente: una parte del territorio de un Estado aspira a dejar de pertenecer a la Unión Europea. El antiguo artículo 299 (que regulaba esta cuestión) no ofrecía cobertura para tal próposito. Dicho precepto, como el actual, preveía que los Estados aumentaran o disminuyeran su territorio sin intervención de la Unión Europea, pero para lo que de ningún modo los habilitaba, era y es, para excluir unilateralmente una parte de su territorio del ámbito espacial de la Unión Europea.

Establecido lo anterior podemos concluir ya que, desde el punto de vista del territorio, existen importantes diferencias entre la Unión Europea y los esquemas federales. Dentro de estos últimos, la delimitación del territorio es siempre una competencia federal (artículo 4 de la Constitución de EE. UU o artículo 23 de la Constitución alemana). En el caso de la Unión Europea, por el contrario, se trata de una competencia exclusiva de los Estados miembros.

\subsection{Las competencias relativas a la nacionalidad y a la ciudadanía}

Lo que vamos a decir sobre este otro elemento constitutivo del Estado, la población, o dicho con otras palabras, sobre el ámbito de aplicación personal del derecho comunitario, no difiere mucho de lo que hemos ya expuesto respecto del territorio. Se trata de una problemática que fue analizada y debatida intensamente con ocasión de la introducción de la noción de "ciudadanía europea" por el Tratado de la

14 HaRTLEY, T. C.: Constitutional problems of the European Union, Hart, Oxford/Portland, 1999, pp. 163 y ss. 
Unión Europea de 1992. Al fin y al cabo, la ciudadanía se configura como la más alta manifestación posible del elemento personal de cualquier comunidad política. En relación con ella el dato clave nos lo proporciona el artículo 8 del TICE: "Toda persona que ostente la nacionalidad de un Estado miembro posee la ciudadanía de la Unión, que se añade a la ciudadanía nacional sin sustituirla». Nos encontramos así con que, al igual que ocurriera con el territorio, la ciudadanía europea es definida por remisión a lo que dispongan los Estados miembros para su propia nacionalidad.

La determinación, por tanto, de quienes sean ciudadanos europeos sigue siendo una competencia exclusiva de los Estados miembros. La Unión Europea no limita en modo alguno a los Estados a la hora de regular su nacionalidad respectiva, pero sí que les impone el deber de reconocer incondicionalmente la nacionalidad otorgada por cualquier otro Estado miembro. En este sentido resulta rotunda la afirmación contenida en la Declaración núm. 2 del Tratado de la Unión Europea de 1992: "La cuestión de saber si una persona posee una nacionalidad determinada se resolverá únicamente remitiéndose al derecho nacional del Estado miembro que se trate». Por ello, en el ámbito de la Unión Europea, y frente a la regla vigente en el Derecho Internacional General, ninguna institución comunitaria, ni ningún Estado miembro pueden objetar la nacionalidad otorgada por otro. La jurisprudencia del Tribunal de Justicia ha confirmado reiteradamente esta tesis desde su conocida sentencia Micheletti de 7 de julio de 1992.

Establecido lo anterior podemos afirmar que, respecto a la población, también son evidentes las diferencias existentes entre la Unión Europea y los esquemas federales. Dentro de estos últimos, las reglas relativas a la nacionalidad se ubican siempre en el ámbito de competencia federal. En el caso de la Unión Europea, por el contrario, se trata de una competencia exclusiva de los Estados miembros.

Dicho esto, conviene no obstante subrayar que, aunque el poder de los Estados para regular la nacionalidad es absoluto, éstos se han visto privados de la facultad de objetar la nacionalidad otorgada por otro, facultad que les vendría conferida por el Derecho Internacional General. Según el Derecho Internacional, desde el ya clásico pronunciamiento del Tribunal Internacional de Justicia en el Ilamado asunto Nottebohm de 6 de abril de 1955, corresponde a cada Estado soberano regular su propia nacionalidad, y sobre los demás Estados recae la obligación de reconocer aquélla. Ahora bien, ese deber de reconocimiento no es absoluto, ya que un Estado puede desconocer legítimamente una nacionalidad otorgada por otro cuando ésta sea ficticia. Esto quiere decir que 
según el Derecho Internacional Público hoy vigente un Estado no está obligado a reconocer la nacionalidad otorgada por otro si ésta no está basada en un vínculo real, suficiente y efectivo entre la persona y el Estado.

Pues bien, en el ámbito de la Unión Europea, los Estados miembros se encuentran privados de esa facultad de objetar nacionalidades ficticias. En la Unión Europea los Estados miembros no pueden, en ningún caso, objetar la nacionalidad conferida por otro.

El estudio comparado de la jurisprudencia del Tribunal Internacional de Justicia (doctrina Nottebohm) y del Tribunal de Justicia de las Comunidades Europeas (doctrina Micheletti) ${ }^{15}$, nos pone así de manifiesto que las normas relativas a la ciudadanía y la nacionalidad, no encajan en el esquema federal, pero se han apartado ya de la solución puramente internacional.

Establecido lo anterior, procede examinar el contenido concreto, esto es el conjunto de derechos específicos que el TICE atribuye al estatuto de ciudadanía europea. Está recogido en el apartado segundo del citado artículo 8. Según él, los ciudadanos de la Unión tienen los siguientes derechos: a circular y residir libremente en el territorio de los Estados miembros; de sufragio activo y pasivo en las elecciones al Parlamento europeo y en las elecciones municipales del Estado miembro en el que residan, en las mismas condiciones que los nacionales de dicho Estado; de acogerse, en el territorio de un tercer país en el que no esté representado el Estado miembro del que no sean nacionales, a la protección de las autoridades diplomáticas y consulares de cualquier Estado miembro en las mismas condiciones que los nacionales de dicho Estado; a formular peticiones al Parlamento Europeo, a recurrir al Defensor del Pueblo Europeo, y a dirigirse a las instituciones y organismos consultivos de la Unión en una de las lenguas de la Constitución y a recibir una contestación en esa misma lengua.

A lo dispuesto en este artículo hay que añadir como principal innovación del TICE, la inclusión en la Parte Segunda del mismo de la Carta de los Derechos Fundamentales de la Unión.

15 Un sugerente comentario de la Sentencia relativa al Asunto Nottebohm (TIJ, Sentencia de 6 de abril de 1955) en PuENTE EgIDO, J.: Casos prácticos de Derecho Internacional Público, 3. ${ }^{\text {a }}$ ed., Imprenta Kadmos, Salamanca, 2000, pp. 295-306. Un estudio comparado de ambas sentencias desde una perspectiva crítica en TCHIKAYA, B.: Mémento de la jurisprudence de droit international public, Hachette, París, 2000, pp. 71 y ss. 
Desbordaría los límites de este trabajo el análisis de todos y cada uno de los mencionados derechos por lo que únicamente voy a subrayar el significado y alcance de esa inclusión así como sus limitaciones.

Desde su inclusión en 1992 a propuesta del gobierno socialista español, la noción de "ciudadanía europea" representa un avance cualitativo importante, porque supone la atribución a los nacionales de los Estados miembros de derechos políticos sin ninguna conexión con las competencias socioeconómicas de las Comunidades Europeas. Por primera vez "se contempla en las normas primarias del derecho comunitario una atribución de derechos no vinculada al ámbito de la competencia material del Tratado sino al hecho de ser ciudadano de la Unión Europea» ${ }^{16}$.

En este sentido, la Carta de Derechos Fundamentales incluida en la Parte Segunda del TICE incluye los mencionados derechos de ciudadanía entre los derechos de todos los individuos de la U.E., con un contenido casi idéntico, excepción hecha del derecho a una buena administración, en el que se incluyen el derecho de acceso del público a los documentos y el derecho a dirigirse a las instituciones comunitarias, junto con el derecho a ser oído en caso de adopción de medidas individuales desfavorables, la obligación de motivación de las decisiones y el derecho a obtener reparación por daños y perjuicios ocasionados por la administración comunitaria.

La atribución a la Carta, como parte integrante del TICE, de valor jurídico vinculante, ha sido una de las razones en que se ha basado la defensa del cáracter constitucional de aquél. Ahora bien, frente a esto hemos de decir que la inclusión en el TICE de las dos dimensiones del principio político liberal inherente a toda Constitución auténtica (separación de los poderes y garantía de los derechos) no es suficiente para conferirle el carácter de Constitución en la medida en que el texto no es producto de la aplicación del principio democrático basado en la titularidad del Poder Constituyente por parte del Pueblo.

Pero, a mayor abundamiento, y sin negar el avance que supone la extensión del derecho de sufragio pasivo y activo en las elecciones municipales y europeas, en la mencionada tabla de derechos no figuran los derechos políticos por antonomasia, la facultad de participar en los asuntos públicos mediante el sufragio en aquellas elecciones en que se designan a los representantes nacionales o regionales que han de

16 LiÑAn Nogueras, D. J.: "La ciudadanía de la Unión Europea» en Rodriguez Iglesias, G. C. y Liñán Nogueras, D.J. (dirs.) El derecho comunitario europeo y su aplicación judicial, Madrid, 1993, p. 272. 
conformar la voluntad del Estado. La titularidad de esos derechos sigue reservada en exclusiva a los nacionales (en España por imperativo constitucional, art. 13 C.E.). Los Estados miembros conservan en el TICE la competencia exclusiva para determinar la titularidad y el ejercicio de los derechos políticos, por lo que, también desde esta óptica, la Unión se aleja de los esquemas federales donde la mencionada facultad es de titularidad estatal. En ello ha visto el profesor Ruipérez "el último y desesperado intento de preservar la soberanía de los Estados» ${ }^{17}$.

\subsection{Las competencias relativas al uso de la coacción}

Fueron Max Weber y Hans Kelsen los más cualificados y lúcidos exponentes de una corriente teórica, todavía vigente, según la cual el rasgo definitorio del Estado moderno viene dado por el monopolio del uso legítimo de la violencia ${ }^{18}$.

El Estado federal asume ese rasgo y en la medida en que se configura como un auténtico Estado, dispone de medios para, llegado el caso, ejercer la coacción. A diferencia de lo que ocurriera en la Confederación, el Estado Federal ejerce directamente su poder sobre los particulares y puede, igualmente, asegurar la ejecución forzosa de sus actos por parte de las entidades federadas. Dicho con otras palabras, la coacción reviste dos dimensiones, de los poderes públicos frente a los particulares, y, dentro de los propios poderes públicos, del uso de la fuerza del todo frente a las partes. Por lo que a nuestro tema interesa baste poner de manifiesto que ninguna de esas dos facetas de la coacción se dan en la Unión Europea. Por el contrario ambas se manifiestan en todos los Estados miembros.

a) Por lo que se refiere a la coacción sobre los particulares, la Unión carece de instrumentos coactivos y depende siempre de los Es-

17 A lo que ha añadido con gran realismo y sagacidad lo siguiente: «Ahora bien, si esto es así, lo que cabría preguntarse, y yo sólo lo apunto, es si realmente puede sostenerse que la soberanía estatal está a salvo cuando, como consecuencia de la integración económica, todas o casi todas las riquezas de ese Estado están en mano de las grandes trasnacionales europeas". RUIPEREZ, J.: La Constitución europea..., ob. cit., p. 81. Dicho con otras palabras: ¿Qué sentido tiene privar a un ciudadano comunitario residente en España del derecho de sufragio en las elecciones generales y permitirle simultáneamente hacerse con el control de empresas estratégicas o de importantes medios de comunicación?

18 Weber, M.: Economía y Sociedad, Fondo de Cultura Económica, México, D.F., 1992, pp. 1047 y ss. KelSEN, H.: Teoría General del Derecho y del Estado, UNAM, México, D.F., 1979, pp. 17 y ss. 
tados miembros. Así, el artículo III-307 del TICE prevé que «la ejecución forzosa se regirá por las normas de procedimiento civil vigentes en el Estado miembro en cuyo territorio se lleve a cabo. La orden de ejecución será consignada, sin más control que el de la comprobación de la autenticidad del título, por la autoridad nacional que el Gobierno de cada uno de los Estados miembros designe a tal efecto (...) Cumplidas estas formalidades a instancia del interesado, éste podrá promover la ejecución forzosa conforme al Derecho interno, recurriendo directamente a la autoridad competente".

b) Por otro lado, no existe en el ámbito de la Unión Europea, nada parecido a la posibilidad de que la Unión reprima por la fuerza aquellos comportamientos de los Estados miembros que supongan una amenaza o impliquen un atentado contra la unidad de la Unión o contra sus principios fundamentales. Ante comportamientos de este tenor, el Tratado únicamente prevé la suspensión de determinados derechos inherentes a la pertenencia a la Unión. Tal es el tenor del artículo 58 del TICE que lleva por rúbrica "Suspensión de los derechos de pertenencia a la Unión».

"1. El Consejo de Ministros, por mayoría de cuatro quintas partes de sus miembros, a propuesta motivada de un tercio de los Estados miembros, del Parlamento Europeo o de la Comisión y previa aprobación del Parlamento Europeo, podrá adoptar una decisión europea en la que constate la existencia de un riesgo claro de violación grave por parte de un Estado miembro de los valores enunciados en el artículo 2. Antes de proceder a esta constatación, el Consejo de Ministros oirá al Estado miembro de que se trate y por el mismo procedimiento podrá dirigirle recomendaciones. El Consejo de Ministros comprobará de manera periódica si los motivos que han llevado a tal constatación siguen siendo válidos. 2. El Consejo Europeo, a propuesta de un tercio de los Estados miembros o de la Comisión y previa aprobación del Parlamento Europeo, podrá adoptar por unanimidad una decisión europea en la que constate la existencia de una violación grave y persistente por parte de un Estado miembro de los valores enunciados en el artículo 2, tras invitar a dicho Estado miembro a que presente sus observaciones. 3 . Cuado se haya efectuado la constatación prevista en el apartado 2, el Consejo de Ministros podrá adoptar, por mayoría cualificada, una decisión europea que suspenda determinados derechos derivados de la aplicación de la Constitución al Estado miembro de que se trate, incluidos los derechos de voto del Estado miembro en el Consejo de Ministros. Al proceder a dicha suspensión, el Consejo de Ministros tendrá en cuenta las posibles consecuencias para los derechos y obligaciones de las personas físicas y jurídicas. El Estado miembro de que se trate seguirá, en cualquier caso, vinculado por las obligaciones que le incumben en virtud de la Constitución». 
Este precepto resulta de una importancia decisiva. De él cabe deducir, con claridad, que la Unión Europea se basa en la renuncia a ejercer la fuerza sobre los Estados miembros. En todo caso, y dejando al margen los problemas que la aplicación de estas disposiciones indudablemente han de provocar, por lo que a nuestro tema interesa baste subrayar que no existe en el TICE nada parecido a la institución de la ejecución federal, esto es, la posibilidad de hacer cumplir sus obligaciones por la fuerza a los Estados miembros.

\subsection{La facultad de abandonar la Unión Europea}

EI TICE no contempla, expresamente, la posibilidad de expulsión de un Estado miembro pero sí regula en el artículo 59 la retirada voluntaria de la Unión. Artículo éste, innecesario es recordarlo, que no encuentra equivalente en ninguna Constitución del mundo, y que confirma la naturaleza convencional y no constitucional de la Unión:

"1. Todo Estado miembro podrá decidir, de conformidad con sus normas constitucionales, retirarse de la Unión Europea. 2. El Estado miembro que decida retirarse notificará su intención al Consejo Europeo, que dará curso a dicha notificación. A la vista de las orientaciones del Consejo Europeo, la Unión negociará y celebrará con ese Estado un acuerdo que regulará la forma de su retirada, teniendo en cuenta el marco de sus relaciones futuras con la Unión. El Consejo de Ministros celebrará ese acuerdo en nombre de la Unión por mayoría cualificada, previa aprobación del Parlamento Europeo. El representante del Estado miembro que se retire no participará ni en las deliberaciones ni en las decisiones del Consejo Europeo o del Consejo de Ministros que le afecten. La Constitución dejará de aplicarse al Estado de que se trate a partir de la fecha de entrada en vigor del acuerdo de retirada o, en su defecto, a los dos años de la notificación prevista en el apartado 2, salvo si el Consejo Europeo, de acuerdo con dicho Estado, decide prorrogar dicho plazo».

Recapitulando lo expuesto hasta ahora podemos afirmar que, los Estados miembros, aunque han transferido múltiples y relevantes competencias a la Unión Europea, (entre las cuales por su relevancia práctica y simbólica, destaca la política monetaria) siguen conservando los atributos básicos inherentes a la idea misma de Estado ${ }^{19}$, como una unidad de acción y de decisión política, y son estos atributos los que en definitiva nos obligan a diferenciar a la Unión Europea de un auténtico Estado Federal. Como hemos expuesto, los Estados miembros, siempre

19 Díez PicAzo, L. M.: "¿Qué significa ser Estado...", ob. cit., p. 283. 
que respeten los valores y principios vertebradores de la Democrcia Constitucional, pueden adoptar el sistema político que estimen conveniente; los Estados miembros continuan delimitando su propio territorio y definiendo su propia nacionalidad, y de esta forma, determinan y definen también el territorio y la ciudadanía de la Unión Europea; sólo los Estados, y no la Unión, están provistos de instrumentos de fuerza coactiva; finalmente, los Estados conservan la decisiva facultad de abandonar la Unión.

\section{LA NATURALEZA JURÍDICA DEL TICE: UN TRATADO INTERNACIONAL}

Con las anteriores premisas estamos ya en condiciones de responder al interrogante básico de si el TICE es en verdad una nueva Constitución europea en virtud de la cual nacería una unión de Derecho Constitucional en la que los Estados miembros quedarían disueltos por haber renunciado a su condición de soberanos, o si, por el contrario, su naturaleza jurídica sigue siendo la de un tratado internacional que viene a modificar el sistema institucional y normativo de la Unión, pero manteniendo ésta como unión contractual, de Derecho Internacional.

$\mathrm{Si}$, como hemos visto, los Estados miembros conservan las facultades y competencias inherentes a su condición de Estados soberanos y, en consecuencia, la Unión Europea que el TICE instituye se configura como una Confederación de Estados en su forma moderna, evidente resulta que el Tratado no puede ser calificado de constitucional, salvo que admitamos la posibilidad de establecer una "Constitución confederal». Posibilidad ésta que descartaremos en el último epígrafe de nuestra exposición.

La Unión Europea como Confederación de Estados en su forma moderna, presenta un elemento común con la versión antigua de la misma, y este no es otro que ser una Unión de Derecho Internacional y no de Derecho Constitucional. Dicho con otras palabras, la naturaleza del vínculo contractual o el fundamento de la organización misma no es, ni puede ser, una Constitución sino que es un Tratado Internacional, que difiere de otros por su contenido (acto fundacional) pero no por su naturaleza.

En todo caso, las dudas que puedan existir sobre el particular se disipan por completo si examinamos por una parte el proceso de elaboración y aprobación del TICE, y por otra, los procedimientos en él previstos para su reforma. El estudio de ambos extremos va a confirmar 
nuestra tesis de que el texto que nos ocupa es un Tratado y no una Constitución.

\subsection{El procedimiento de elaboración del TICE}

A diferencia de los anteriores Tratados, que habían sido negociados en el seno de Conferencias intergubernamentales en las que sólo participaron representantes de los gobiernos de los Estados miembros, el PTICE fue elaborado por una Convención, creada al efecto ${ }^{20}$, y en la que estaban representados intereses diversos. Con independencia de las inevitables connotaciones revolucionarias del término "Convención", es preciso subrayar que los miembros de la misma no fueron reclutados mediante elección popular.

La Convención estaba compuesta por un Presidente y dos Vicepresidentes, quince representantes de los jefes de Estado o de gobierno de los Estados miembros (1 por Estado), 13 representantes de los jefes de Estado o de gobierno de los países candidatos a la adhesión (1 por país); 30 representantes de los parlamentos nacionales de los Estados miembros (2 por Estado); 26 representantes de los parlamentos nacionales de los Estados candidatos (2 por país); 16 representantes miembros del Parlamento Europeo y 2 representantes de la Comisión Europea.

Nos encontramos pues que frente a lo acaecido en el pasado, el PTICE ha sido elaborado por un colegio en el que han participado representantes no sólo de los gobiernos sino también de los Parlamentos, incluidos los de los Estados candidatos. Ahora bien, en modo alguno se puede calificar a esta Convención como constituyente. $Y$ ello por varias razones.

En primer lugar, la Convención no es un órgano que actúe en nombre del Pueblo. Ni en nombre del Pueblo europeo porque no existe, ni en el de los Pueblos de los Estados miembros. Si lo hiciera, resultaría inaceptable, desde la más elemental lógica política democrática, admitir que sus decisiones pudieran resultar alteradas por las voluntades de los integrantes de la Conferencia Intergubernamental. Y es justamente esto último lo que está previsto y lo que ha ocurrido.

20 Similar procedimiento se siguió para elaborar la Carta de Derechos Fundamentales de la Unión Europea, que fue solemnemente proclamada por los Presidentes del Parlamento Europeo, del Consejo de la Unión Europa y de la Comisión Europea, el 7 de diciembre de 2000, en el marco del Consejo Europeo de Niza. 
Ello está en relación con la segunda razón que, a nuestro juicio, impide calificar a la Convención como constituyente. La Convención no es la autora del Tratado, es un mero órgano encargado de preparar un primer proyecto de Tratado que será negociado, como en el pasado, en el seno de una CIG. Es, por tanto, finalmente este órgano el que adopta la versión definitiva del Proyecto, que una vez fijado, habrá de ser sometido a los procedimientos de ratificación previstos en cada uno de los ordenamientos constitucionales de los Estados miembros. Para decirlo con mayor claridad y contundencia, la CIG puede disponer con absoluta y plena libertad del Texto aprobado por la Convención. La Convención ha podido debatir sobre todo, proponer lo que quiera, pero no decidir nada.

Desde una perspectiva formal el texto del TICE emanará de un proceso de reforma derivado del actual artículo 48 TUE, y por tanto, no puede tratarse sino de un Tratado Internacional ${ }^{21}$.

\subsection{El procedimiento de revisión del TICE}

El análisis del procedimiento previsto en el TICE para su reforma nos confirma también, una vez más, que nos encontramos ante un auténtico Tratado Internacional.

Como ha recordado la profesora García Gestoso en su meritorio y sugerente estudio sobre el particular, durante la celebración de la CIG del año 2000 que alumbró el Tratado de Niza, "se fue más consciente que nunca de los problemas de alcanzar acuerdos unánimes entre los Estados para modificar los Tratados comunitarios»"22. Los múltiples pro-

\footnotetext{
21 MARTIN Y PÉREZ de NANClARES, J.: «El proyecto de Constitución europea: reflexiones sobre los trabajos de la Convención" en Revista de Derecho Comunitario Europeo, n. ${ }^{\circ} 15,2003$, p. 530. La explícita referencia a una Constitución europea «no conlleva en el fondo un avance constitucional sustancial. A nuestro entender, contribuye simplemente a proyectar la naturaleza mixta de la Unión y a clarificar el grado de integración ya alcanzado anteriormente. No crea un nuevo orden jurídico ni rompe con el proceso anterior. Se limita a simplificar, consolidar y actualizar lo conseguido hasta el momento". Ibid, p. 531.

22 García Gestoso, N.: El proceso de revisión en el Proyecto del Tratado por el que se instituye una Constitución para Europa. Presentado al VIII Congreso Iberoamericano de Derecho Constitucional (Sevilla, Diciembre 2003). Ejemplar mecanografiado, p. 17. De la misma autora, resulta también de interés y sumamente esclarecedor para nuestro tema el siguiente trabajo: "Algunas cuestiones sobre la soberanía en el proceso de integración europea» en Revista de Derecho Político, n. ${ }^{\circ} 57,2003$, pp. 157-198.
} 
blemas que rodearon la génesis del mencionado Tratado pusieron de relieve la imposibilidad de seguir avanzando a través de los sistemas de reforma previstos para modificar los Tratados constitutivos.

Ahora bien, a pesar de ello, el análisis de los trabajos de la Convención nos pone de manifiesto que la Parte IV en la que se regula el procedimiento de revisión, fue la que más tardíamente se abordó. Igualmente, los debates, las enmiendas planteadas y los distintos textos que se sucedieron muestran cómo, pese a que se plantearon alternativas distintas, nunca existió un propósito real de modificar el régimen tradicionalmente existente. ${ }^{23}$

En este sentido, el TICE regula el procedimiento de revisión en su artículo IV-7 en los siguientes términos:

«1. El Gobierno de cualquier Estado miembro, el Parlamento Europeo o la Comisión podrán presentar al Consejo de Ministros proyectos de revisión del Tratado por el que se instituye la Constitución, que se notificará a los parlamentos nacionales de los Estados miembros.

2. Si el Consejo Europeo, previa consulta al Parlamento Europeo y a la Comisión, adopta por mayoría simple una decisión favorable al examen de las modificaciones propuestas, el Presidente del Consejo Europeo convocará una Convención compuesta por representantes de los parlamentos nacionales de los Estados miembros, del Parlamento Europeo y de la Comisión. En el caso de modificaciones institucionales en el ámbito monetario se consultará también al Banco Central Europeo. El Consejo Europeo podrá decidir por mayoría simple, previa aprobación del Parlamento Europeo, no convocar la Convención en caso de modificaciones cuya importancia no lo justifique. En este último caso, el Consejo Europeo establecerá un mandato para la Conferencia de los representantes de los Gobiernos de los Estados miembros.

La Convención examinará los proyectos de revisión y adoptará por consenso una recomendación a la Conferencia de los representantes de los gobiernos de los Estados miembros prevista en el apartado 3.

3. La Conferencia de los representantes de los Gobiernos de los Estados miembros será convocada por el Presidente del Consejo de Ministros, con el fin de que se aprueben de común acuerdo las modificaciones del Tratado por el que se instituye la Constitución.

Las enmiendas entrarán en vigor después de haber sido ratificadas por todos los Estados miembros, de conformidad con sus respectivas normas constitucionales.

${ }^{23}$ García Gestoso, N.: «El proceso de revisión...» ob. cit., p. 20. Algunos miembros de la Convención plantearon la necesidad de superar la unanimidad para la revisión. 
4. Si, transcurrido un plazo de dos años desde la firma del Tratado por el que se instituye la Constitución, las cuatra quintas partes de los Estados miembros lo hubieran ratificado y uno o varios Estados miembros hubieran experimentado dificultades para proceder a dicha ratificación, el Consejo Europeo tomará conocimiento de la cuestión".

Varias son las novedades que este precepto introduce respecto al procedimiento de reforma anteriormente existente. Ahora bien, ninguna de ellas resulta sustancial y ello por la razón evidente de que la férrea regla de la unanimidad estatal, el predominio intergubernamental y la remisión a la ratificación por parte de todos los Estados miembros se mantiene ${ }^{24}$.

Entre las novedades procedimentales cabe señalar las siguientes: a) En primer lugar, la adopción de la decisión de abrir el proceso de reforma que antes correspondía al Consejo de Ministros se atribuye ahora al Consejo Europeo, y se establece expresamente que la decisión se adopte por mayoría simple; b) En segundo lugar, se introduce la convocatoria de una Convención para preparar la CIG, con lo que se institucionaliza la práctica seguida para elaborar el PTICE. Por lo que se refiere a la composición, adopción de acuerdos por consenso y valor de sus trabajos, tampoco cambia nada respecto a la práctica anterior. Unicamente se prevé la posibilidad de prescindir de tal convocatoria si las modificaciones propuestas son de esacasa importancia, extremo éste que habrá de decidir el Consejo Europeo previa aprobación del Parlamento Europeo; c) Finalmente, novedosa es la disposición contenida en el apartado cuarto ${ }^{25}$ en el sentido de precisar que el Consejo Europeo analizará la cuestión de si transcurridos dos años desde la firma del TICE, uno o varios Estados tuvieran dificultades para ratificarlo. Cabe señalar el carácter superfluo e innecesario de este apartado. Evidente resulta que el Consejo Europeo analizará ese problema lo diga o no el TICE, tan evidente como que el Consejo Europeo carece de facultades para resolver un problema que aun afectando a la Unión, depende exclusivamente de los Estados miembros: la ratificación.

Procede ahora comparar el procedimiento descrito con los procesos de reforma de la Constitución del Estado Federal y de los Tratados fundacionales de las Confederaciones, para verificar si nos encontramos en el primero o en el segundo de los supuestos contemplados.

24 García Gestoso, N.: "El proceso de revisión...», ob. cit., p. 21.

${ }_{25}$ Esta cláusula se reproduce literalmente en la Declaración al Acta Final de firma del TICE para su entrada en vigor. 
a) El procedimiento de reforma del pacto contractual de la Confederación arcaica. El pacto fundacional de las Confederaciones es un Tratado Internacional basado en un acuerdo libre y voluntario entre Estados soberanos. La preservación de la soberanía de los Estados miembros exige que para cualquier reforma de ese pacto originario se exija como requisito inexcusable el consentimiento unánime de todos los Estados miembros de la Confederación. Ello se plasma jurídicamente bien en el derecho de veto que impide la reforma bien en el derecho a separarse de la confederación si no se acepta la modificación ${ }^{26}$. Estas afirmaciones vienen avaladas por el examen de las tres Confederaciones que la Historia y el Derecho comparados nos presentan: la Confederación norteamericana (1777-1787), la Confederación helvética (1803-1848), y la Confederación germánica (1815-1871).

b) En el procedimiento de reforma de la Constitución del Estado Federal concurren siempre estas dos características: la necesaria participación en el proceso tanto de las colectividades miembros como de la organización política central, y la aplicación de la regla de la mayoría de los Estados miembros para proceder a la aprobación de la reforma. Ello se traduce en la inexistencia de derecho de veto alguno por parte de las colectividades miembros y en la imposibilidad jurídica de llevar a cabo una secesión del Estado por no estar conforme con la reforma constitucional verificada.

Confrontado el contenido del artículo IV-7 del TICE con los modelos históricos de referencia, nos vemos obligados a incluirlo en el primer grupo. También desde esta perspectiva, la del procedimiento de reforma, el TICE se configura como un auténtico Tratado Internacional ${ }^{27}$.

26 «La Confederación descansa sobre un pacto en el que subsiste el carácter contractual y que no puede, por consiguiente, ser modificado en contra de uno cualquiera de los Estados sin su consentimiento, expresado por sus órganos superiores, sea que la revisión exige el acuerdo de todos los Estados, sea que, pudiendo ser hecha por el acuerdo de la mayoría, simple o reforzada, ahora cada uno de los discrepantes tiene una facultad jurídica de secesión". DuRAND, $\mathrm{CH}_{\text {.: }}$ Confédération d'Etats et Etat Etat Fédéral. Realisation acquisis et perspectives nouvelles, Paris, 1955, p. 20, tomo la cita de RUIPÉREZ, J.: La «Constitución europea...», ob. cit., p. 163.

27 A mayor abundamiento, y como ya hemos expuesto, el TICE ha incluido en el Título IX de su Parte I, la previsión de la retirada voluntaria de un Estado de la Unión, que habrá de adoptarse conforme con sus normas constitucionales para proceder seguidamente a negociarse un acuerdo entre el Estado que se retira y la Unión. En nombre de ésta celebrará el acuerdo el Consejo de Ministros por mayoría cualificada. Como agudamente ha observado la profesora García Gestoso en su 
El alcance de lo expuesto hasta ahora no es puramente teórico ${ }^{28}$. Antes al contrario, la afirmación de que el TICE pese a su ambiguo y contradictorio nombre es en realidad un Tratado Internacional y no una Constitución, implica una serie de consecuencias de orden prácti$\mathrm{co}^{29}$. La principal de ellas, la consideración de los Estados como sujetos que sustentan el completo proceso de integración, lo que hace de ellos los únicos y auténticos "señores de los Tratados». El presente y el futuro de la Unión Europea depende de lo que decidan los Estados que la integran y que, como expusimos páginas atrás, aunque han transferido múltiples facultades y competencias a la Unión Europea, conservan las competencias inherentes a su condición de Estados en tanto que unidades de acción y decisión soberanas.

Dicho esto, el TICE sólo podría ser considerado como una auténtica Constitución de la Unión Europea en la medida en que veamos en él una "Constitución confederal». En los fecundos y meritorios trabajos del profesor La Pérgola encontramos la más brillante formulación de esa tesis. Sin embargo, por las razones que vamos a exponer, no podemos compartirla.

\section{SOBERANÍA Y DEMOCRACIA EN LA UNIÓN EUROPEA: ¿UNA CONSTITUCIÓN CONFEDERAL?}

EI TICE no implica en modo alguno la disolución de los Estados miembros de la Unión en la nueva comunidad política. Comunidad que hemos calificado como Confederación de Estados en su forma moderna.

trabajo antes citado, ello quiere decir que jurídicamente resulta más fácil abandonar la Unión que reformarla. Como fue advertido por bastantes miembros de la Convención, esta posibilidad representa una seria amenaza a esgrimir en cualquier procedimiento de reforma.

28 MAtíA, J.: "¿Hay una Constitución europea?», en VV. AA. La democracia constitucional. Libro Homenaje al Prof. Rubio Llorente. Madrid, 2002, vol. II, pp. 1361 y ss.

${ }_{29}$ Aunque desborda el objeto de este trabajo, es obligado subrayar que entre esas consecuencas ocupa un lugar destacado la posición del órgano de representación de los Estados en el procedimiento de adopción de decisiones. Frente a la utópica pretensión de equiparar el Parlamento Europeo a un Parlamento nacional, hay que reconocer que ello supondría relegar a un segundo plano en el proceso decisorio europeo a los sujetos principales del mismo. Esto es lo que determina que, inevitablemente, el Consejo de Ministros y el Consejo Europeo ocupen una posición privilegiada en la estructura de la Unión. En este sentido, el TICE culmina el largo camino recorrido afirmando, sin ambages, el principio político de doble legitimidad (ciudadanos y Estados) que se plasma y traduce, jurídicamente, en el procedimiento de codecisión entre el Parlamento y el Consejo. 
Se trata de una Confederación que presenta un grado de centralización mucho más avanzado que lo que estuvieron nunca las Confederaciones históricas. Los destinatarios de las normas dictadas por los poderes centrales no son únicamente las autoridades de las colectividades federadas, sino también los ciudadanos. Ahora bien pese a ese mayor grado de centralización, la Confederación moderna "está destinada a no convertirse, en ningún caso, en un ente estatal ${ }^{30}$. "La Confederación -escribe el profesor La Pergola- adquiere nuevas potencialidades y aplicaciones funcionales: no es ya sólo como en el pasado un débil y precario ordenamiento que precedía al Estado federal, sino que puede constituir el definitivo punto de llegada de un proceso de integración» ${ }^{31}$.

Desde esta óptica, el profesor La Pergola considera que las normas convencionales, esto es, los Tratados Internacionales, no son los instrumentos adecuados para regular el proceso político de la Confederación moderna y ello por la sencilla razón de que la experiencia histórica demuestra que toda Confederación arcaica fundada en un Tratado Internacional acabó, bien transformada en un auténtico Estado Federal, bien condenada a su disolución.

Por todo ello, y refiriéndose por tanto a la Confederación moderna, el ilustre jurista italiano afirma: "Ha pasado el tiempo de las uniones fundadas sobre un Tratado entre soberanos e indiferentes a los grandes temas de los que se ocupa una Constitución, que debe desatar el espinoso nudo de las relaciones entre el hombre y su gobierno" ${ }^{32}$. En este contexto surge la idea de una "Constitución confederal»"33, esto es, de poner en marcha en el ámbito de la Unión Europea una Constitución racional normativa. Constitución que no sería ya el resultado de un pacto contractual entre Estados soberanos, sino la expresión de la voluntad soberana de un auténtico Poder Constituyente.

Por ello, y desde una perspectiva rigurosamente democrática, el maestro italiano afirma: "La Constitución (europea) debe emanar del poder constituyente, poder éste que aún no se le ha reconocido a la Asamblea de Estrasburgo" ${ }^{34}$. Pero que, de acuerdo con la lógica cons-

30 La Pergola, A.: Los nuevos senderos..., ob. cit., p. 133.

31 La Pergola, A.: Los nuevos senderos..., ob. cit., p. 171.

32 La Pergola, A.: Los nuevos senderos..., ob. cit., p. 188.

33 Sobre los antecedentes de esta idea, RuIPÉREZ, J.: La Constitución europea..., ob. cit., pp. 160 y 161. El concepto de "Constitución confederal» lo encontramos en la clasificación de las Constituciones llevada a cabo por Wheare atendiendo al modelo de Estado a que dan origen. WhEARE, K. C.: Las Constituciones modernas, Barcelona, 1975, 2 a $^{\text {ed., }}$ pp. 24 y ss.

34 La Pergola, A.: Los nuevos senderos..., ob. cit., p. 183. 
titucional habrá que concedérsele si de verdad se quiere aprobar una Constitución democrática. Según la tradición constitucional europea, que difiere en esto de la norteamericana, la potestad constituyente puede delegarse, por lo que el Parlamento que elaborase la Constitución recibiría de los ciudadanos un poder especial para ello. El texto elaborado por esta Asamblea Constituyente debería después ser ratificado por el Pueblo europeo en un referéndum convocado al efecto.

En este contexto, ninguna duda debe albergarse sobre el carácter auténticamente constitucional del Texto Fundamental así elaborado y aprobado. Ahora bien, la sugerente tesis del profesor La Pergola presenta un eslabón débil, que acertada y agudamente ha sido puesto de manifiesto por el profesor Ruipérez. La aprobación por el Parlamento Europeo de un Texto Constitucional, escribe el Catedrático de la Universidad de La Coruña, "tanto si operamos con las formulaciones clásicas de Wise o Isnard, como si lo hacemos desde los esquemas propios del constitucionalismo democrático y social, requerirá como condición previa la realización del pacto social, por el cual esos ciudadanos europeos se invisten como Pueblo soberano de la nueva entidad jurídico-política» ${ }^{35}$.

En similares términos se pronuncia el profesor Grimm. El insigne jurista alemán sostiene que es inherente a una Constitución en el sentido pleno del término el que «se refiera a un acto realizado por, o al menos atribuido a, el pueblo en sí, y conforme al cuál éste se atribuye capacidad política a sí mismo" ${ }^{36}$. Lo cual no ocurre en la Unión Europea en la medida en que los Estados miembros siguen siendo los autores de los Tratados. Por ello, el poder público europeo «no es el que se deriva del pueblo, sino un poder en el que median los Estados $"{ }^{37}$.

En mi opinión, el pacto social por el cual surgiría el nuevo Poder Constituyente, podría verificarse tanto en el momento de celebración de las elecciones constituyentes al Parlamento Europeo como en el del referéndum de ratificación del Texto Constitucional aprobado por aquél. Pero lo que resulta indiscutible es su necesidad como prius lógico de toda Constitución democrática. El pacto social precede siempre al acto constitucional. Por el primero se crea la sociedad, mediante el segundo se organiza. Sin la existencia de un poder constituyente previo y residenciado además en el Pueblo no cabe hablar de Constitución democrática.

35 RuIPÉREZ, J.: La Constitución europea..., ob. cit., p. 166.

36 Grimm, D.: “Does Europe need a Constitution?» en ELJ, 1, 1995, p. 282.

37 Grimm, D.: Does Europe...,ob. cit., p. 291. 
Y es aquí donde como bien advierte el profesor Ruipérez las tesis del profesor italiano incurren en una contradicción que con todos los respetos para quien puede ser considerado uno de los grandes maestros del Derecho Público de nuestro tiempo, debemos denunciar. Tras haber admitido la necesidad de un Poder Constituyente del Pueblo Europeo, entiende La Pergola que "la actividad de la Unión Europea debe conjugar el principio de la soberanía popular con el de la soberanía de los Estados miembros ${ }^{38}$. Ello quiere decir que una vez que se ha aprobado la Constitución europea la condición de soberano debe reconocerse tanto al Pueblo Europeo en su conjunto como a cada uno de los Pueblos de los Estados miembros individualmente considerados ${ }^{39}$. La Constitución confederal nos conduce así a la clásica tesis de la doble soberanía o cosoberanía ${ }^{40}$. Ahora bien, aunque como muy bien advierte el profesor Ruipérez la finalidad perseguida ahora por La Pergola con su tesis de la cosoberanía es muy distinta a la que inspiró a Hamilton o a Madison, sus consecuencias son las mismas por lo que las críticas que históricamente se formularon a las primeras son plenamente aplicables a la última. El propósito del profesor italiano resulta claro: "La apelación al mantenimiento de los Estados miembros en la condición de soberanos -escribe Ruipérez- se explica ahora como el último intento para mantener a la Unión Europea en el estadio confederal ${ }^{41}$. Ahora bien, la tesis de que en un sistema federal se produce un reparto o división de la soberanía entre la organización central y las colectividades miembros, se opone frontalmente a la idea misma de soberanía que, como poder absoluto e ilimitado, es por definición siempre, única e indivisible. Así lo expusieron con claridad y acierto Rousseau $^{42}$, Jellinek ${ }^{43}$ o Heller ${ }^{44}$. Por ello, la tesis de la cosoberanía se nos

38 La Pergola, A.: Los nuevos senderos..., ob. cit., p. 186.

39 RUIPÉREZ, J.: La Constitución europea..., ob. cit., p. 166.

40 Formulada originariamente por Hamilton y Madison, HAMILTON, A., MADISON, J., y JAY, J.: El federalista, FCE, México, D.F. 1994 (Quinta reimpresión), pp. 12-15, 32-35, 123-129 y $158-163$.

41 RuIPÉREZ, J.: La Constitución europea..., ob. cit., p. 168.

42 «Por la misma razón que la soberanía no es enajenable es indivisible; porque la voluntad es general o no lo es; es la del cuerpo del pueblo o solamente de una parte de él. En el primer caso, esta voluntad declarada es un acto de soberanía y hace ley; en el segundo, no es sino una voluntad particular o un acto de magistratura: es a lo más un decreto". Rousseau, J. J.: Contrato social (1762), Traducción de Fernando de los Ríos (1921) y Prólogo de M. Tuñón de Lara, Espasa Calpe, Madrid, 5. ${ }^{\mathrm{a}}$ ed. revisada, 1990, pp. 58-60.

43 «El fundamento de la concepción jurídica del Estado está constituido por el reconocimiento de éste como de una unidad; de donde se sigue, como consecuencia necesaria, la doctrina de la indivisibilidad del poder del Estado. Un poder dividido, 
presenta en realidad como un expediente para intentar eludir el problema nuclear que subyace en todo debate sobre la constitucionalización de Europa, y que con mejor o peor fortuna estas páginas han pretendido afrontar, y que no es otro que el relativo a si los Estados miembros como Estados soberanos e independientes subsisten o se han disuelto en la Unión. "Porque la existencia de dos sujetos soberanos en un mismo territorio es (...) absolutamente irrealizable desde el punto de vista de la ontología del Estado mismo - escribe Ruipérezde lo que se trata es de determinar si la soberanía pertenece al Pueblo Europeo, como entidad política unitaria, o, por el contrario, aquélla reside en cada uno de los Pueblos de los diversos Estados que componen la Unión Europea» ${ }^{45}$. Las consecuencias de una u otra alternativa no pueden resultar más diferentes. Ahora bien, como vamos a ver, ninguna de las dos hipótesis conduce a una Constitución confederal.

La primera hipótesis, esto es, la atribución de la soberanía al Pueblo Europeo, implica que se ha verificado el pacto social, bien en el momento de las elecciones a la Asamblea constituyente bien en el de la celebración del referendum constitucional. Ahora bien, lo que no puede ni debe obviarse son las consecuencias que, de manera tan necesaria como inevitable, el pacto social implica. El nacimiento del Pueblo europeo como Poder constituyente determina que en él «se integrarán y se disolverán los Pueblos de los actuales Estados miembros, y que, en cuanto titular de la soberanía, puede imponer su voluntad a cada uno de los que lo integran ${ }^{46}$. Solamente en este supuesto cabe entender que el Texto Fundamental de la Unión Europea es una Constitución auténtica, pero no una Constitución confederal, sino la Constitución de un Estado Federal Europeo ${ }^{47}$.

supone el desmenuzamiento del Estado en una variedad de formaciones políticas. Lo que se dice respecto del poder del Estado, vale también, como es natural, para cuanto se refiera al poder soberano del mismo". JELLINEK, G.: Teoría General del Estado. Traducción y prólogo de la segunda edición alemana por Fernando de los Ríos. Comares, Granada, 2000, p. 488.

44 «Es posible que dos ejércitos luchen por establecer sus respectivas soberanías sobre un territorio determinado, en cuyo caso, el jurista tendrá que aceptar la existencia de una lucha por la soberanía, que durará hasta la terminación de la guerra. Es en cambio imposible aceptar que sobre un mismo territorio existan dos unidades decisorias supremas; su existencia significaría la destrucción de la unidad del Estado y su consecuencia sería el estallido de la guerra civil». HELLER, H.: La soberanía. Contribución a la teoría del derecho estatal y del derecho internacional, Tra-

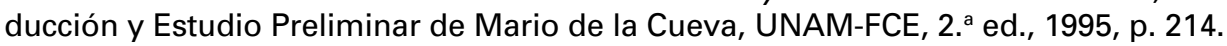

45 RUIPÉREZ, J.: La Constitución europea..., ob. cit., p. 170.

46 RUIPÉREZ, J.: La Constitución europea..., ob. cit., pp. 170-171.

47 Grimm, D.: Does Europe..., ob. cit., pp. 298-299. 
La segunda hipótesis basada en la conservación de la soberanía por todos y cada uno de los Pueblos de los Estados miembros, supone que no se ha verificado pacto social alguno por lo que al no haber surgido un Poder Constituyente resulta absolutamente imposible hablar de una Constitución en el sentido racional normativo o liberal burgués que manejamos en nuestra exposición ${ }^{48}$. El Texto Fundamental de la Unión Europa será, en este segundo supuesto, un Tratado Internacional que establece una Confederación de Estados en su forma moderna.

El examen de los procedimientos de elaboración y de reforma del TICE y el análisis de su contenido por lo que se refiere al reparto competencial entre los Estados y la Unión nos confirma claramente que nos encontramos ante la segunda alternativa. El hipotético sometimiento del TICE a un referendum europeo no alteraría esta situación. En primer lugar porque el referendum no es suficiente para legitimar un texto que no ha sido hecho en nombre del pueblo ni con un mandato expreso para ello, pero, sobre todo y aunque no quisiera admitirse lo anterior, porque su contenido, como he tratado de exponer con mayor o menor acierto, consagra el principio del respeto a la identidad nacional de los Estados miembros que conservan las facultades inherentes a su condición de soberanos, incluida la de retirarse voluntariamente de la Unión.

En definitiva, y como ha subrayado el profesor Haberle, no puede hablarse de una Constitución europea «toda vez que Europa como tal no forma un único Estado Constitucional» ${ }^{49}$.

Y abundando en nuestra argumentación, debemos añadir que ni siquiera estamos asistiendo a un proceso constituyente europeo. Dicho proceso sólo podrá considerarse abierto con la celebración de unas

48 Todo edificio constitucional debe construirse sobre dos pilares o fundamentos: el principio político democrático según el cual el Poder Constituyente reside en el Pueblo y el principio jurídico de supremacía constitucional que es consecuencia del anterior. Dicho con otras palabras, la Constitución debe configurarse como norma suprema porque es obra del Poder Constituyente. La gran paradoja del TICE, —que por lo demás no es una novedad, sino que se limita a plasmar un principio de creación jurisprudencial vigente desde hace décadas-, es que consagra el principio de su supremacía normativa. Pero dicho principio jurídico no puede fundamentarse en el principio democrático. Por decirlo con mayor claridad y contundencia, aunque el TICE no es obra del Pueblo Europeo, sus disposiciones prevalecerán sobre los Textos Constitucionales emanados de todos y cada uno de los Pueblos de Europa. Quede así planteada la controvertida cuestión relativa a la legitimidad de una tal supremacía.

49 Haberle, P.: "Derecho Constitucional común europeo», en Revista de Estudios Políticos, n. ${ }^{\circ} 79$, 1993, pp. 11 y ss. 
elecciones constituyentes, esto es, delegando en el Parlamento Europeo, mediante mandato popular expreso, la facultad de elaborar un Texto Fundamental. Solución ésta que nada tiene que ver con el procedimiento de reforma ya analizado y previsto en el propio TICE.

En este sentido cualquier analogía entre el significado y alcance de la Convención que ha elaborado el proyecto de TICE y cuya intervención se prevé igualmente para ulteriores reformas del mismo con una Convención constituyente como la de Filadelfia resulta forzada y necesariamente sólo puede inducir al error. Como ha expuesto el profesr Ruipérez, cuando en la Convención de Filadelfia los representantes de los distintos Pueblos de los Estados se ponen de acuerdo en la necesidad de profundizar y perfeccionar la unión y, en consecuencia, acuerdan crear una única comunidad, "lo que en realidad están haciendo no es sino renunciar a su propia individualidad como Pueblos diferenciados, para integrarse en una unidad política superior y única: el Pueblo de los Estados Unidos ${ }^{50}$.

De esta forma la titularidad de la soberanía pasó de los ciudadanos de las Colonias, que dejaron de existir como colectividades independientes, al Pueblo de la nueva comunidad política. Ello permitió que los Founding Fathers, actuando en nombre de esta última elaboraran y discutieran el texto constitucional que después fue establecido y sancionado por el Pueblo de los Estados Unidos como titular indiscutido e indiscutible de la soberanía. Lo que se tradujo jurídicamente en el celebérrimo "We the People... del Preámbulo de la Constitución norteamericana.

En el estadio actual de la integración europea no se ha decidido en modo alguno que los pueblos de los Estados en ella representados renuncien a su individualidad. Ello explica que el TICE no utilice ninguna expresión parecida al "Nosotros, el Pueblo de la Unión Europea ${ }^{51}$ y que, por el contrario, establezca con claridad un principio de doble legitimidad, ("los ciudadanos y los Estados de la Unión Europea"), que se proyectará después, con total coherencia, en el sistema institucional y en el procedimiento decisorio de la Unión.

La Unión Europea sigue siendo, pues, una "Unión de Estados» según la expresión acuñada por el Tribunal Constitucional alemán en su

50 RuIPÉREZ, J.: La Constitución europea..., ob. cit., p. 158.

51 "La constatación es obvia: -escribe Araceli Mangas - no hay un pueblo europeo, sino pueblos de los Estados miembros, y por ello, la Unión Europea sigue teniendo una naturaleza internacional insoslayable». MANGAS, A.: "Reflexiones sobre el proyecto de Constitución europea ante la perspectiva de la reforma de 1996» en Revista Española de Derecho Constitucional, n. ${ }^{\circ} 45,1995$, p. 145. 
sentencia de 12 de octubre de 1993 sobre el Tratado de Maastricht ${ }^{52}$. O como con mayor precisión ha señalado el profesor Rubio Llorente, una Unión de "Estados integrados», poniendo así de manifiesto cómo la pertenencia a la Unión Europea, que, sin duda, implica notables limitaciones de la libertad de los Estados miembros, no anula su condición última de Estados soberanos e independientes ${ }^{53}$.

Mientras el "poder público europeo" se fundamente en los Estados miembros, carece de sentido hablar de Constitución europea. Solamente si dicho poder derivara directamente del Pueblo europeo, cabría hablar en la Unión de legitimidad constitucional. Ahora bien, como muy bien ha advertido el profesor Grimm, en ese supuesto, los Estados miembros habrían dejado de ser los dueños del Tratado y la Unión Europea se habría transformado en un auténtico Estado ${ }^{54}$.

52 TORSTEN, S.: «La sentencia del Tribunal Constitucional alemán sobre el Tratado de Maastricht" en Revista de Instituciones Europeas, n. ${ }^{\circ} 3,1994$, pp. 745 y ss. ALAEZ CORRAL, B.: "Comentario a la STC alemán de 12 de octubre de 1993" en Revista Española de Derecho Constitucional, n. ${ }^{\circ} 45,1995$, pp. 243 y ss.

53 Rubio Llorente, F.: "El constitucionalismo de los Estados integrados en Europa» en Revista Española de Derecho Constitucional, n. ${ }^{\circ} 48,1996$.

54 Grimm, D.: Does Europe..., ob. cit., pp. 298-299. 